\title{
Time-Domain Finite-Element Modeling of Thin Electromagnetic Shells
}

\author{
Johan Gyselinck, Ruth V. Sabariego, Patrick Dular and Christophe Geuzaine
}

\begin{abstract}
In this paper the authors propose a novel timedomain extension of the well-known frequency-domain thinshell approach. The time-domain interface conditions at the shell surface are expressed in terms of the average (zero-order) instantaneous flux and current density vectors in the shell, as well as in terms of a limited number of higher-order components. The method is elaborated for a magnetic vector potential finiteelement formulation. The validation is done by means of two 2-D test cases with pulsed magnetic field excitation. The results are in excellent agreement with those produced by a brute-force model in which the shell is meshed finely throughout its thickness.
\end{abstract}

Index Terms-Electromagnetic shielding, finite element methods, magnetodynamics

\section{INTRODUCTION}

The finite-element (FE) analysis of electromagnetic problems involving thin shells may suffer from meshing difficulties and prohibitive computational cost, depending on the shell thickness compared to both the penetration depth and the two other dimensions of the shell. The well-known thin-shell approach allows to do away with those problems, but it is limited to linear and time-harmonic analyses [1] [2] [3]. These limitations are circumvented in [4] by considering a separate 1-D FE discretisation for each node of the thin-shell surface.

In this paper we propose the use of dedicated polynomial basis functions to account for the variation of flux and current density throughout the shell thickness. The method is elaborated considering a general eddy-current problem comprising a thin shell and validated by means of 2-D test cases.

\section{PROBLEM DEFINITION}

The Maxwell equations relevant to low-frequency eddycurrent problems are

$$
\operatorname{curl} h=j, \quad \operatorname{div} b=0, \quad \operatorname{curl} e=-\partial_{t} b .
$$

We further consider the classical constitutive laws relating the magnetic field $h$ to the flux density (or induction) $b$, and the electric field $e$ to the current density $j$ :

$$
b=\mu h \quad \text { and } \quad j=\sigma e,
$$

where $\mu$ is the permeability and $\sigma$ the conductivity. For the sake of brevity we will consider in the following linear (and isotropic) media only.

The calculation domain $\Omega$ in the Euclidean space comprises an inductor domain $\Omega_{i}$, a conducting and massive region $\Omega_{c}$ and a thin-shell region $\Omega_{s}$ (Fig. 1). The thin shell is magnetic

Manuscript received June 24, 2007. This work was partly supported by the Belgian Science Policy (IAP P6/21).

J. Gyselinck is with the Dept. of Bio-, Electro- and Mechanical Systems (BEAMS), Université Libre de Bruxelles, Belgium (e-mail: johan.gyselinck@ulb.ac.be). R. V. Sabariego, P. Dular and C. Geuzaine are with the Dept. of Electrical Engineering and Computer Science, Institut Montefiore, University of Liège, Belgium. P. Dular is with the Belgian National Fund for Scientific Research (F.N.R.S.). and/or conducting; its thickness will be denoted by $d$. The current density $j$ is known a priori in $\Omega_{i}$, whereas it constitutes an unknown quantity in $\Omega_{c}$ and $\Omega_{s}$.

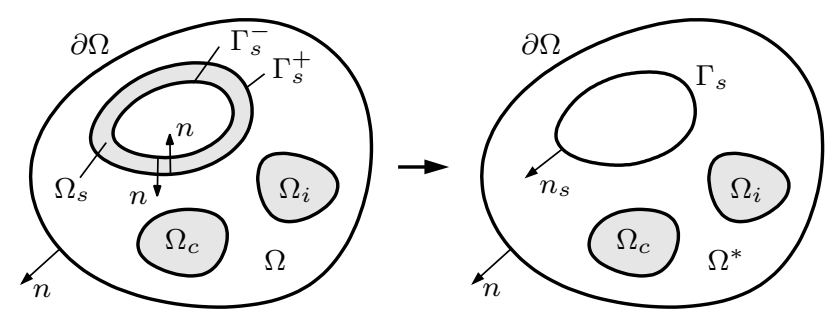

Fig. 1. Calculation domain $\Omega$ and reduction of the thin-shell domain $\Omega_{s}$ to the surface $\Gamma_{s}$

Adopting a magnetic vector potential $a$, with $b=\operatorname{curl} a$ and $e=-\partial_{t} a$, equations (1b) and (1c) are satisfied. By considering test functions $a^{\prime}$ and treating the thin-shell domain $\Omega_{s}$ in the same way as $\Omega_{c}$, one derives the following weak form from the Ampère law (1a):

$$
\begin{aligned}
\left(\nu \operatorname{curl} a, \operatorname{curl} a^{\prime}\right)_{\Omega}+ & \left(\sigma \partial_{t} a, a^{\prime}\right)_{\Omega_{c} \cup \Omega_{s}}+ \\
& \left\langle n \times h, a^{\prime}\right\rangle_{\partial \Omega}=\left(j, a^{\prime}\right)_{\Omega_{i}},
\end{aligned}
$$

where $(\cdot, \cdot)_{\Omega}$ and $\langle\cdot, \cdot\rangle_{\partial \Omega}$ are the integrals on the domain $\Omega$ and on the boundary $\partial \Omega$ respectively of the product of the two (scalar or vector) arguments; $n$ is the outward normal on $\partial \Omega$; $\nu=1 / \mu$ is the reluctivity.

The domain $\Omega$ and the weak form (3) can be discretized by Whitney edge elements, leading to a system of linear firstorder differential equations in terms of the degrees of freedom of the magnetic vector potential [3]. Note that the latter has to be gauged in order to make it unique. For a frequency-domain calculation, considering sinusoidal quantities of pulsation $\omega$ and adopting the complex formalism, (3) leads to a system of complex algebraic equations.

The thin-shell approach consists in reducing the volume region $\Omega_{s}$ to an "average" surface $\Gamma_{s}$ (situated "half way" between the inner surface $\Gamma_{s}^{-}$and the outer surface $\Gamma_{s}^{+}$of $\Omega_{s}$, and with outward normal $n_{s}$ ) and modifying the weak form (3) on the basis of a 1-D thin-shell model. The latter model will be developed in the next section III, first in the frequency domain and then in the time domain. In section IV, the timedomain 1-D thin-shell model will be incorporated in the 3-D FE analysis.

\section{1-D THIN-SHELL MODEL}

In the 1-D model of the shell, the variation of the component of $h, b, e$ and $j$ tangential to $\Gamma_{s}$ is considered throughout the thickness. (The variation in the two other dimensions of the shell is not considered, and neither is the normal component of these four fields.) In particular, the tangential components of the magnetic and electric fields on either surface of the shell 
(i.e. on $\Gamma_{s}^{+}$and $\Gamma_{s}^{-}$) are defined as

$$
\begin{array}{ll}
h_{t}^{+}=n_{s} \times\left(\left.h\right|_{\Gamma_{s}^{+}} \times n_{s}\right), & h_{t}^{-}=n_{s} \times\left(\left.h\right|_{\Gamma_{s}^{-}} \times n_{s}\right), \\
e_{t}^{+}=n_{s} \times\left(\left.e\right|_{\Gamma_{s}^{+}} \times n_{s}\right), & e_{t}^{-}=n_{s} \times\left(\left.e\right|_{\Gamma_{s}^{-}} \times n_{s}\right) .
\end{array}
$$

\section{A. Governing differential equations}

We adopt a local coordinate system $x, y, z$ with the $z$-axis normal to the shell (i.e. parallel to $n_{s}$ ) and with $z=0$ situated in the middle of the shell, and consider the following vector quantities tangential to $\Gamma_{s}: h_{t}(z, t), b_{t}(z, t), e_{t}(z, t)$ and $j_{t}(z, t)$. The 1-D eddy-current problem in the shell $(-d / 2 \leq$ $z \leq d / 2)$ is then governed by the following two equivalent partial differential equations:

$$
\begin{array}{rll}
\partial_{z}^{2} h_{t}=\sigma \partial_{t} b_{t} & \text { with } & h_{t}(z, t)=\nu b_{t}(z, t), \\
\partial_{z}^{2} e_{t}=\mu \partial_{t} j_{t} & \text { with } & e_{t}(z, t)=\rho j_{t}(z, t),
\end{array}
$$

where $\rho=1 / \sigma$ is the resistivity.

The associated boundary conditions are

$$
\begin{aligned}
h_{t}^{+} & =h_{t}(d / 2, t), & & h_{t}^{-}(t)=h_{t}(-d / 2, t), \\
e_{t}^{+} & =e_{t}(d / 2, t), & & e_{t}^{-}(t)=e_{t}(-d / 2, t) .
\end{aligned}
$$

Essential global quantities are the average flux density vector $b_{0}(t)$ and the average current density vector $j_{0}(t)$ :

$$
b_{0}(t)=\frac{1}{d} \int_{-d / 2}^{d / 2} b_{t}(z, t) d z, j_{0}(t)=\frac{1}{d} \int_{-d / 2}^{d / 2} j_{t}(z, t) d z,
$$

which are both tangential to $\Gamma_{s}$.

\section{B. Decomposition in even and odd parts}

It will prove useful to separate the boundary conditions (89) and the ensuing solution of (6-7) in an even and an odd part with respect to $z$. These components are indicated with the superscripts $e$ and $o$, respectively:

$$
\begin{aligned}
h^{e}(t) & =\frac{h_{t}^{+}+h_{t}^{-}}{2}, & h^{o}(t) & =\frac{h_{t}^{+}-h_{t}^{-}}{2}, \\
e^{e}(t) & =\frac{e_{t}^{+}+e_{t}^{-}}{2}, & e^{o}(t) & =\frac{e_{t}^{+}-e_{t}^{-}}{2},
\end{aligned}
$$

with

$$
\begin{aligned}
h_{t}^{+}=h^{e}+h^{o}, & h_{t}^{-}=h^{e}-h^{o}, \\
e_{t}^{+}=e^{e}+e^{o}, & e_{t}^{-}=e^{e}-e^{o} .
\end{aligned}
$$

1) Net flux and zero net current (Fig. 2): The even part of $b_{t}(z, t)$ produces a nonzero $b_{0}(t)$, whereas the corresponding odd $j_{t}(z, t)$ leads to $j_{0}(t)=0$. The Faraday law (1c) gives

$$
e^{o}(t)=n_{s} \times \frac{d}{2} \partial_{t} b_{0}
$$

For a sinusoidal time variation at pulsation $\omega$, we define the relative shell thickness $d^{*}$ on the basis of the penetration depth $\delta$ :

$$
d^{*}=d / \delta \quad \text { with } \quad \delta=\sqrt{2 / \sigma \mu \omega} .
$$

The analytical frequency-domain resolution of (6) leads to following equation in terms of the complex representation (symbols in bold) of $h^{e}(t)$ and $b_{0}(t)$ [1]:

$$
\boldsymbol{h}^{e}=\nu \boldsymbol{Y}\left(d^{*}\right) \boldsymbol{b}_{0},
$$

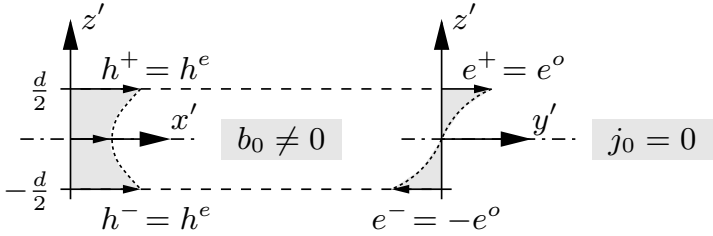

Fig. 2. Symmetry with nonzero flux and zero current (with $h_{t}(z, t)$ along $x$ and $e_{t}(z, t)$ along $y$, for instance)

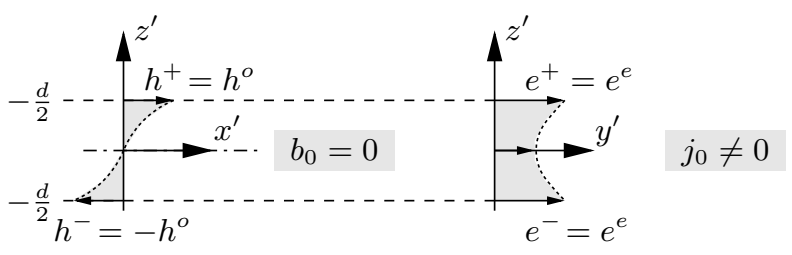

Fig. 3. Symmetry with nonzero current and zero flux (with $h_{t}(z, t)$ along $x$ and $e_{t}(z, t)$ along $y$, for instance)

with

$$
\boldsymbol{Y}\left(d^{*}\right)=\frac{1+\boldsymbol{i}}{2} d^{*} \operatorname{cotanh}\left(\frac{1+\boldsymbol{i}}{2} d^{*}\right),
$$

where $i$ is the imaginary unit. At low frequency, $0<d^{*} \ll 1$, $\boldsymbol{Y}$ tends towards 1; at sufficiently high frequency, say $d^{*}>6$, $\boldsymbol{Y}$ is practically equal to $\frac{1+i}{2} d^{*}$.

2) Net current and zero net flux (Fig. 3): The even part of $j_{t}(z, t)$ produces a nonzero $j_{0}(t)$, whereas the corresponding odd $b_{t}(z, t)$ leads to $b_{0}(t)=0$. The Ampère law (1a) gives

$$
h^{o}(t)=-n_{s} \times \frac{d}{2} j_{0}(t) .
$$

Analogous to (17) we have

$$
\boldsymbol{e}^{e}=\rho \boldsymbol{Y}\left(d^{*}\right) \boldsymbol{j}_{0} .
$$

\section{Time-domain extension with dedicated basis functions}

The equations (11-14), $(15,19)$ and $(17,20)$ allow to include the 1-D thin-shell model in a 3-D FE analysis; this amounts to the well-known frequency-domain approach [1] [2] [3]. The time-domain extension of the latter approach requires the time-domain extension of $(17,20)$. These equations concern the even component of $b_{t}(z, t)$ (net flux) and the even component of $j_{t}(z, t)$ (net current), respectively. Their time-domain extension can be achieved by considering a number of even, orthogonal polynomial basis functions $\alpha_{k}(z)$ [5].

We develop in detail the symmetric case with net flux. The induction vector $b_{t}(z, t)$ being then an even function of $z$, it can be approximated as the following truncated series:

$$
b_{t}(z, t)=\sum_{k=0,2, \ldots}^{n} \alpha_{k}(z) b_{k}(t),
$$

where $\alpha_{0}(z)=1, \alpha_{2}(z)=-1+12 z^{2} / d^{2}, \alpha_{4}(z)=3-$ $120 z^{2} / d^{2}+560 z^{4} / d^{4}, \ldots$ are orthogonal and have unit value on either surface of the shell $\left(\alpha_{k}( \pm d / 2)=1\right)$.

Satisfying the differential equation (6a) strongly, the magnetic field $h_{t}(z, t)$ can be written as

$$
h_{t}(z, t)=h^{e}(t)+\sigma d^{2} \sum_{k=0,2, \ldots}^{n} \beta_{k+2}(z) \partial_{t} b_{k}(t),
$$

where the even polynomial functions $\beta_{k+2}(z)$ satisfy the following equations:

$$
d^{2} \partial_{z}^{2} \beta_{k+2}=\alpha_{k}(z) \quad \text { and } \quad \beta_{k+2}( \pm d / 2)=0 .
$$


We then enforce the constitutive law (6b) weakly by means of the $n / 2+1$ functions $\alpha_{k}(z)(k=0,2, \ldots, n)$ :

$$
\int_{-d / 2}^{d / 2} \alpha_{k}(z)\left(h_{t}(z, t)-\nu b_{t}(z, t)\right) d z=0 .
$$

The resulting system of $n / 2+1$ first-order differential equations in terms of the induction vector components can be written as follows:

$$
\left[H^{e}(t)\right]=\nu[P]\left[B_{0: n}(t)\right]+\sigma d^{2}[Q] \partial_{t}\left[B_{0: n}\right],
$$

with $\left[H^{e}\right]=\left[\begin{array}{lllll}h^{e} & 0 & 0 & \ldots & 0\end{array}\right]^{T},\left[\begin{array}{ll}B_{0: n}\end{array}\right]=\left[\begin{array}{lllll}b_{0} & b_{2} & b_{4} & \ldots & b_{n}\end{array}\right]^{T}$, and $[P]$ and $[Q]$ square matrices of order $n / 2+1$. Thanks to the orthogonality of the basis functions $\alpha_{k}$ and (23), $[P]$ and $[Q]$ are diagonal and symmetric tridiagonal, respectively. In the following the nonzero elements of $[P]$ and $[Q]$ will be denoted by $p_{k}$ and $q_{k, l}$, with $k, l=0,2, \ldots n$. For the case $n=4$ these elements are: $p_{0}=1, p_{2}=1 / 5, p_{4}=1 / 9$, $q_{00}=1 / 12, q_{22}=1 / 210, q_{44}=1 / 1386, q_{02}=q_{20}=-1 / 60$ and $q_{24}=q_{42}=-1 / 1260$.

Analogously, for the electric field and the current density vectors we find

$$
\left[E^{e}(t)\right]=\rho[P]\left[J_{0: n}(t)\right]+\mu d^{2}[Q] \partial_{t}\left[J_{0: n}\right],
$$

with $\left[E^{e}\right]=\left[\begin{array}{lllll}e^{e} & 0 & 0 & \ldots & 0\end{array}\right]^{T},\left[\begin{array}{ll}J_{0: n}\end{array}\right]=\left[\begin{array}{lllll}j_{0} & j_{2} & j_{4} & \ldots & j_{n}\end{array}\right]^{T}$, and $[P]$ and $[Q]$ the same matrices as in (25).

Considering an imposed sinusoidal $h^{e}(t)$ of frequency $f$ (with relative shell thickness $d^{*}$ ), the steady-state solution $b_{0}(t)$ of (25) leads to an approximation $\boldsymbol{Y}^{(n)}\left(d^{*}\right)=\boldsymbol{h}_{e} /\left(\nu \boldsymbol{b}_{0}\right)$ of the analytical expression $\boldsymbol{Y}\left(d^{*}\right)$ given by (18). The value of $n$ should be chosen in agreement with the desired accuracy in the relevant frequency range. For instance, allowing a maximum relative error of $1 \%$, the approximations $n=0$, $n=2$ and $n=4$ are valid up to roughly $d^{*}$ equal to 1,4 and 8 respectively [5].

\section{FE FORMULATION WITH THIN-SHELL MODEL}

As a first step towards the thin-shell formulation, the thinshell volume $\Omega_{s}$ is excluded from the original calculation domain. As the boundary of the new domain $\Omega \backslash \Omega_{s}$ is augmented with the surfaces $\Gamma_{s}^{+}$and $\Gamma_{s}^{+}$, corresponding surface terms have to be considered in the weak formulation (3), with normal $n$ outward with respect to $\Omega \backslash \Omega_{s}$, as in Fig. 1 (left). Next the surfaces $\Gamma_{s}^{+}$and $\Gamma_{s}^{-}$are slightly moved so as to coincide with the average surface $\Gamma_{s}$ (with outward normal $n_{s}$ ). This leads to the calculation domain $\Omega^{*}$ and the thin-shell surface $\Gamma_{s} \equiv \Gamma_{s}^{*+} \equiv \Gamma_{s}^{*-}$. The weak form now reads

$$
\begin{aligned}
& \left(\nu \operatorname{curl} a, \operatorname{curl} a^{\prime}\right)_{\Omega^{*}}+\left(\sigma \partial_{t} a, a^{\prime}\right)_{\Omega_{c}}+\left\langle n \times h, a^{\prime}\right\rangle_{\partial \Omega} \\
& \quad+\left\langle n_{s} \times h, a^{\prime}\right\rangle_{\Gamma_{s}^{*-}}-\left\langle n_{s} \times h, a^{\prime}\right\rangle_{\Gamma_{s}^{*+}}=\left(j, a^{\prime}\right)_{\Omega_{i}} .
\end{aligned}
$$

For taking into account the time-domain behavior of the thin shell, we introduce the tangential vector fields $b_{0}, b_{2}, \ldots$, $b_{n}$ and $j_{0}, j_{2}, \ldots, j_{n}$ on $\Gamma_{s}$ as unknowns. Since we chose to satisfy (6a) and (7a) in a strong sense and (6b) and (7b) in a weak sense, the discretizations of these fields can be chosen independently. Here, we chose to discretize both using Whitney edge elements, which enables normal discontinuity of the tangential fields within the shell.

From (12b), (15) and $e=-\partial_{t} a$, assuming zero initial conditions for $a$, we verify that the net flux $d b_{0}$ in the shell requires the tangential component of the magnetic vector potential to be discontinuous across $\Gamma_{s}$ :

$$
a_{t}^{+}-a_{t}^{-}=-n_{s} \times d b_{0} .
$$

We therefore decompose the potential $a$ into components $a_{c}$ and $a_{d}$, where $a_{c, t}=n_{s} \times\left(a_{c} \times n_{s}\right)$ and $a_{d, t}=n_{s} \times\left(a_{d} \times n_{s}\right)$ are continuous and discontinuous across the shell, respectively. For $a_{c}$ a conventional discretization with Whitney edge elements throughout $\Omega$ can also be adopted. Without loss of generality we can choose $a_{d}$ to be zero in the volume enclosed by $\Gamma_{s}$. Furthermore, by limiting its support to one layer of elements adjacent to $\Gamma_{s}^{+}$, we can make the discretisation of $a_{d}$ and $-n_{s} \times d b_{0}$ to be conform [3]. In the following we can then simply denote $a_{d}$ by $-n_{s} \times d b_{0}$.

By considering $a^{-}=a_{c}$ and $a^{+}=a_{c}-n_{s} \times d b_{0}$, and by taking into account (11b) and (19), we work out the two new surface terms in (27):

$$
\begin{array}{r}
\left\langle n_{s} \times h, a^{\prime}\right\rangle_{\Gamma_{s}^{*-}}-\left\langle n_{s} \times h, a^{\prime}\right\rangle_{\Gamma_{s}^{*+}} \\
=\left\langle n_{s} \times h_{t}^{-}, a_{c, t}^{\prime}\right\rangle_{\Gamma_{s}}-\left\langle n_{s} \times h_{t}^{+}, a_{c, t}^{\prime}-n_{s} \times d b_{0}^{\prime}\right\rangle_{\Gamma_{s}} \\
=\left\langle n_{s} \times h_{t}^{+}, n_{s} \times d b_{0}^{\prime}\right\rangle_{\Gamma_{s}}-\left\langle n_{s} \times\left(n_{s} \times d j_{0}\right), a_{c, t}^{\prime}\right\rangle_{\Gamma_{s}} \\
=d\left\langle h_{t}^{+}, b_{0}^{\prime}\right\rangle_{\Gamma_{s}}-d\left\langle j_{0}, a_{c, t}^{\prime}\right\rangle_{\Gamma_{s}} .
\end{array}
$$

Considering (13a), (19) and the first equation of (25), with $p_{0}=1$, we can express $h_{t}^{+}$in (29) in terms of $b_{0}, j_{0}$ and $b_{2}$ :

$$
h_{t}^{+}=\nu b_{0}-n_{s} \times \frac{d}{2} j_{0}+\sigma d^{2}\left(q_{0,0} \partial_{t} b_{0}+q_{0,2} \partial_{t} b_{2}\right),
$$

assuming $n \geq 2$. This allows to explicit (27) as a weak form in terms of $a_{c}$ and $b_{0}$ (or $a_{d}$ ) in $\Omega^{*}$, and $b_{0}, b_{2}$ and $j_{0}$ on $\Gamma_{s}$, and with test functions $a_{c}^{\prime}$ and $b_{0}^{\prime}$.

Next, from (12a) and the first equation of (26) we obtain

$$
\rho j_{0}+\mu d^{2}\left(q_{0,0} \partial_{t} j_{0}+q_{0,2} \partial_{t} j_{2}\right)=-\partial_{t} a_{c, t}+n_{s} \times \frac{d}{2} \partial_{t} b_{0},
$$

which we weakly impose on $\Gamma_{s}$ with test functions $j_{0}^{\prime}$.

The remaining equations of the systems (25) and (26) give rise to the following weak forms with test functions $b_{l}^{\prime}$ and $j_{l}^{\prime}$ $(l=2,4, \ldots, n)$ :

$$
\begin{aligned}
& 0=\left\langle\nu p_{l} b_{l}, b_{l}^{\prime}\right\rangle_{\Gamma_{s}}+\sum_{i=-2,0,2}\left\langle\sigma d^{2} q_{l, l+i} \partial_{t} b_{l+i}, b_{l}^{\prime}\right\rangle_{\Gamma_{s}}, \\
& 0=\left\langle\rho p_{l} j_{l}, j_{l}^{\prime}\right\rangle_{\Gamma_{s}}+\sum_{i=-2,0,2}\left\langle\mu d^{2} q_{l, l+i} \partial_{t} j_{l+i}, j_{l}^{\prime}\right\rangle_{\Gamma_{s}},
\end{aligned}
$$

where for $l=n$ the last term $(i=2)$ in (32) and (33) should be ignored.

\section{ApPlication eXAmples}

The thin-shell approach proposed above is validated considering two 2-D application examples (Fig. 4), and with $n$ up to 4 . We adopt the classical 2-D magnetic vector potential formulation with the potential chosen normal to the plane of the calculation domain. No gauging is required. First-order triangular and linear elements are used.

In both test cases a brute-force FE model provides a reference solution. In this FE model the thin shell is considered through a fine surface mesh (domain $\Omega_{s}$ ), the number of layers of elements of which is taken to be $3 \max \left(d^{*}, 1\right)$. Both frequency-domain and time-domain results are compared, where the excitation consists of a sinusoidal current (of frequency $f$ ) and a time-periodic pulsed current (of fundamental frequency $f$ ), respectively. In particular, we look at 

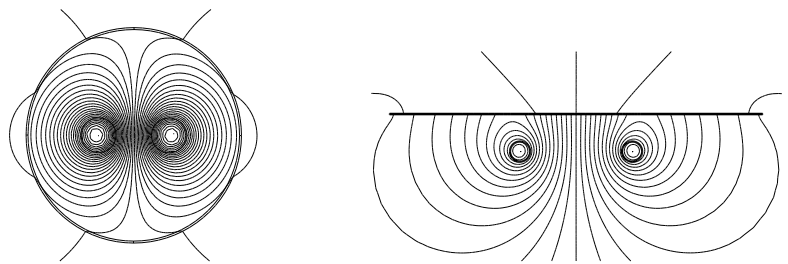

Fig. 4. Left: double line placed inside a nonmagnetic conducting tube and flux lines at $d^{*}=0.2$. Right: magnetic and conducting plate placed above a double line and flux lines at $d^{*}=1$.

the normalized induction magnitude in a given point in the shielded region, where the induction magnitude at peak current and without shielding serves as a base value.

\section{A. Conducting nonmagnetic tube}

The first application example, illustrated in Fig. 4 (left), concerns a double line placed inside a conducting nonmagnetic tube $(d=3 \mathrm{~mm}, \sigma=10 \mathrm{MS} / \mathrm{m}$, inner radius $164 \mathrm{~mm})$. Results for a point situated at $0.5 \mathrm{~m}$ above the tube are shown in Figs. 5 and 6. The pulsed current in Fig. 6 has fundamental period $T=1 / f=98.7 \mu \mathrm{s}\left(d^{*}=2\right)$. Two periods are time stepped from zero initial conditions. The results are quite satisfactory: the solution obtained with the thin-shell approach converges quickly to the reference solution when increasing $n$.

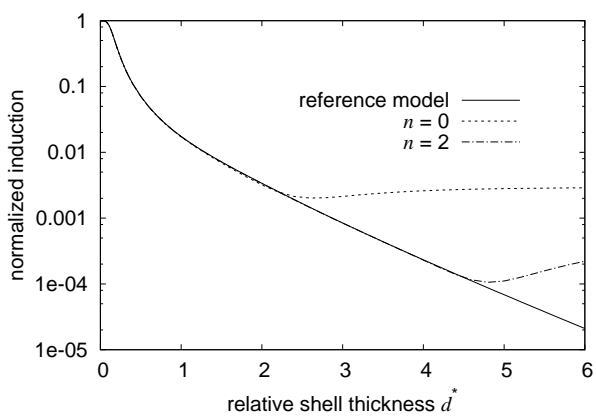

Fig. 5. Normalized amplitude of induction above the tube versus $d^{*}$ in case of sinusoidal current

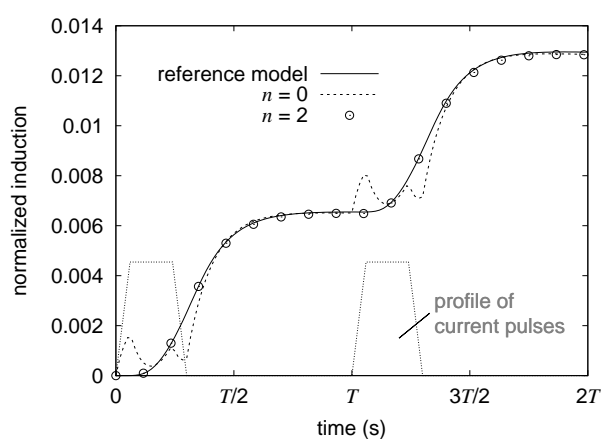

Fig. 6. Normalized induction above the tube in case of pulsed current (with profile indicated)

\section{B. Conducting and magnetic plate}

In the second example the thin shell is a conducting and magnetic plate (length $1 \mathrm{~m}, d=1 \mathrm{~mm}, \sigma=10 \mathrm{MS} / \mathrm{m}, \mu_{r}=$ 1000 ) which is placed above a double line (Fig. 4, right). The results shown in Figs. 7 and 8 concern the induction $0.25 \mathrm{~m}$ above the center of the plate. Excellent convergence is again observed.

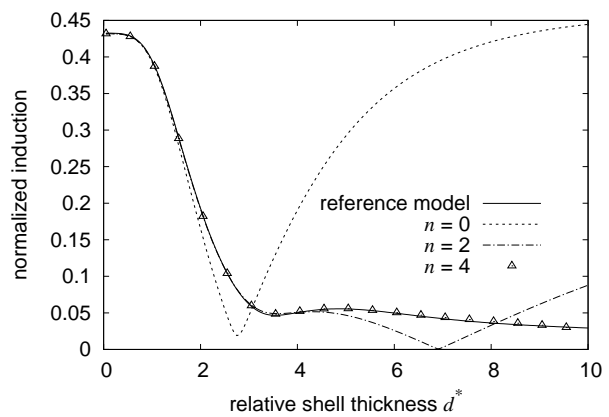

Fig. 7. Normalized amplitude of induction above the plate versus $d^{*}$ in case of sinusoidal current

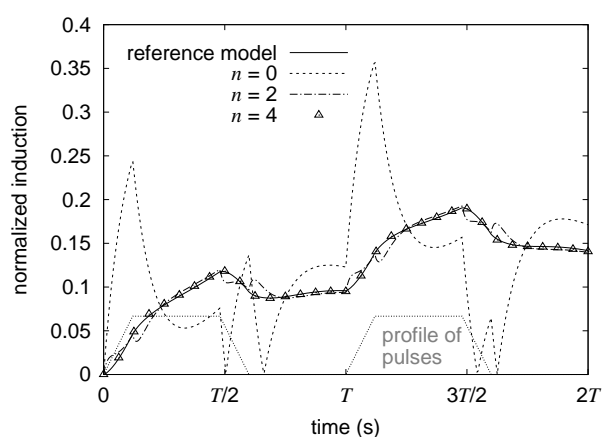

Fig. 8. Normalized induction above the plate versus time in case of pulsed current (with profile indicated, $T=1 / f=2.47 \mathrm{~ms}, d^{*}=4$ )

\section{CONCLUSIONS}

We have proposed a new weak formulation of the timedomain thin-shell eddy-current problem. The unknowns are the magnetic vector potential and a number of flux and current density components on the shell surface. Two application examples have clearly shown that by considering a sufficiently large number of these auxiliary components on the thin shell, a very high accuracy can be achieved. This allows to compromise between accuracy and computational cost. Further, note that the method is straightforwardly extendible to other formulations and to saturable thin shells.

\section{REFERENCES}

[1] L. Krähenbühl and D. Muller, "Thin layers in electrical engineering. Example of shell models in analyzing eddy-currents by boundary and finite elemen methods," IEEE Trans. on Magn., vol. 29, no. 5, pp. 14501455, 1993.

[2] I. D. Mayergoyz and G. Bedrosian, "On calculation of 3-D eddy currents in conducting and magnetic shells," IEEE Trans. on Magn., vol. 31, no. 3, pp. 1319-1324, 1995.

[3] C. Geuzaine, P. Dular, and W. Legros, "Dual formulations for the modeling of thin electromagnetic shells using edge elements", IEEE Trans. on Magn., vol. 36, no. 4, pp. 799-803, 2000.

[4] O. Bottauscio, M. Chiampi, and A. Manzin, "Transient analysis of thin layers for the magnetic field shielding", IEEE Trans. on Magn., vol. 42, no. 4, pp. 871-874, 2006.

[5] J. Gyselinck, R. V. Sabariego, and P. Dular, "A nonlinear time-domain homogenization technique for laminated iron cores in three-dimensional finite element models", IEEE Trans. on Magn., vol. 42, no. 4, pp. 763 766, 2006. 